\title{
ESD as first-intent resection method: also for the duodenum?
}

\section{(1) $\odot \odot$}

\author{
Authors \\ Yutaka Saito, Satoru Nonaka \\ Institution \\ Endoscopy Division, National Cancer Center Hospital, \\ Tokyo, Japan \\ Bibliography \\ DOI https://doi.org/10.1055/a-1012-6455 | \\ Endoscopy International Open 2019; 07: E1761-E1762 \\ (c) Georg Thieme Verlag KG Stuttgart · New York \\ elSSN 2196-9736
}

Kato et al's paper revealed the predictors of technical difficulty of duodenal endoscopic submucosal dissection (ESD), which is regarded as the most challenging current endoscopy technique, and this work deserves great praise for their challenging efforts.

Duodenal ESD is a complicated procedure, even compared to colorectal ESD, which was regarded as most difficult such dissection until now. As a result, it is not a standardized procedure, even in Japan.

The debate between ESD and endoscopic mucosal resection (EMR) for duodenal ESD is similar to that for colorectal EMR and $E S D$, but there are some factors that distinguish duodenal from colorectal ESD:

1. Submucosal cancer is rare, which is different from colorectal laterally spreading tumors.

2. Few recurrences have been reported after a duodenal procedure, even after piecemeal EMR ( $p$-EMR), and p-EMR could show a favorable long-term outcome [1].

3. Risk of intra- and post-procedural perforation is estimated to be very high compared to that for colorectal ESD and severe complications are reported when perforation occurs in duodenal ESD.

Considering these factors together, duodenal ESD is not performed routinely even at National Cancer Center Hospital, Tokyo and EMR or p-EMR is frequently performed for duodenal adenomas.

Cancer risk, however, increases when a duodenal tumor becomes larger, particularly when it is more than half-circumference, and p-EMR is still technically challenging for such huge duodenal tumors. Surgical resection is, therefore, an alternative treatment choice for huge duodenal tumors.
As for surgery, local resection could be less invasive but pancreatoduodenectomy is too aggressive and invasive treatment even for early cancer without risk of lymph-node metastasis. Therefore, ESD has been considered for large duodenal tumors. But in the current study, it proved technically challenging, therefore dictating that it be done only by expert hands or in high-volume centers.

Quite recently, laparoscopy endoscopy cooperative surgery (LECS) [2] has been developed for treatment of upper gastrointestinal stromal tumors (GIST). It combines less-invasive therapy with laparoscopic and endoscopic treatment. LECS is anticipated to be effective and has been used for some large duodenal tumors. A less-invasive, effective and safe strategy such as LECS would be highly sought after for treatment of duodenal tumors.

In the west, full-thickness closure devices such as Overstitch have received an approvement by the US Food and Drug Administration and have been used in duodenal ESD [3]. Another full-thickness device, the over-the-scope clip, also is available and used for endoluminal full-thickness resection for duodenal tumors, with a limitation to tumors measuring $<2$ to $3 \mathrm{~cm}$ in diameter [4-6].

With these technical developments for full-thickness closure and resection emerging in the west rather than Japan, this new treatment strategy will change the common strategy of endoluminal surgery drastically in the near future. Nevertheless, small duodenal adenomas $<2 \mathrm{~cm}$ in diameter could be treated effectively and safely by conventional EMR or using under water EMR technique [7]. Given that, challenging ESD should be avoided by inexperienced endoscopists, considering the technical difficulty and high risk of complications. 


\section{Competing interests}

None

References

[1] Nonaka S, Oda I, Tada K et al. Clinical outcome of endoscopic resection for nonampullary duodenal tumors. Endoscopy 2015; 47: 129135

[2] Hiki N, Yamamoto Y, Fukunaga T et al. Laparoscopic and endoscopic cooperative surgery for gastrointestinal stromal tumor dissection. Surg Endosc 2008; 22: 1729-1735
[3] Granata A, Amata M, Ligresti D et al. Underwater full-thickness resection of a duodenal bulb gastrointestinal stromal tumor with OverStitch defect repair. Endoscopy 2019: doi:10.1055/a-0881-2702

[4] Schmidt A, Meier B, Cahyadi O et al. Duodenal endoscopic full-thickness resection (with video). Gastrointest Endosc 2015; 82: 728-733

[5] Bauder M, Schmidt A, Caca K. Endoscopic full-thickness resection of duodenal lesions-a retrospective analysis of 20 FTRD cases. United European Gastroenterol J 2018; 6: 1015-1021

[6] Kappelle WFW, Backes Y, Valk GD et al. Endoscopic full-thickness resection of gastric and duodenal subepithelial lesions using a new, flatbased over-the-scope clip. Surg Endosc 2018; 32: 2839-2846

[7] Binmoeller KF, Weilert F, Shah J et al. "Underwater" EMR without submucosal injection for large sessile colorectal polyps (with video). Gastrointest Endosc 2012; 752: 1086-1091 